\title{
Rates and Determinants for the Use of Anticoagulation Treatment before Stroke in Patients with Known Atrial Fibrillation
}

\author{
Michela Giustozzi Giancarlo Agnelli Silvia Quattrocchi \\ Monica Acciarresi Andrea Alberti Valeria Caso Maria Cristina Vedovati
}

Michele Venti Maurizio Paciaroni

Stroke Unit and Division of Cardiovascular Medicine, University of Perugia, Santa Maria

della Misericordia Hospital, Perugia, Italy

\section{Keywords}

Atrial fibrillation $\cdot$ Antithrombotic treatment $\cdot$ Stroke $\cdot$ Oral anticoagulants

\begin{abstract}
Introduction and Objective: Even though the introduction of less cumbersome anticoagulant agents has improved, the rates of overall anticoagulant treatment in eligible patients with atrial fibrillation (AF) remain to be defined. We aimed to assess the rates of and determinants for the use of anticoagulation treatment before stroke in patients with known AF since the introduction of direct oral anticoagulants (DOAC) in clinical practice. Methods: Consecutive patients admitted to an individual stroke unit, from September 2013 through July 2019, for acute ischemic stroke or transient ischemic attack (TIA) with known AF before the event were included in the study. Logistic regression analysis was used to identify independent predictors of the use of anticoagulant treatment. Results: Overall, 155 patients with ischemic stroke/TIA and known AF were included in this study. Among 152 patients with a $\mathrm{CHA}_{2} \mathrm{DS}_{2}$-VASc score $>1,43$ patients were not receiving any treatment, 47 patients were receiving antiplatelet agents, and the remaining 62 patients were on oral anticoagulants. Among 34 patients on DOAC, 13 were receiving a nonlabeled reduced dose and 18 out of 34 patients on vitamin $\mathrm{K}$ antagonists had an INR value $<2$ at the time of admission. Before stroke, only 34 out of 155 patients (21.9\%) were adequately treated according to current guidelines. Previous stroke/TIA was the only independent predictor of the use of anticoagulant therapy. Conclusions: Only $21.9 \%$ of the patients hospitalized for a stroke or TIA with known AF before the event were adequately treated according to recent treatment guidelines. It is important to improve medical information about the risk of AF and the efficacy of anticoagulants in stroke prevention.
\end{abstract}




\section{Introduction}

Stroke is a leading cause of mortality and long-term disability worldwide. Every year, more than 795,000 people in the USA have a stroke and nearly 1 stroke out of 4 occur in people who have had a previous stroke [1]. Atrial fibrillation (AF) is an independent risk factor for stroke, leading to a 5-fold increase in the annual risk of stroke [2]. Vitamin K antagonists (VKA) reduce the risk of stroke by $62 \%$ and that of mortality by $26 \%$ with an acceptable risk of bleeding $[3,4]$. Indeed, international guidelines currently recommend oral anticoagulation in all patients with risk factors for stroke [5, 6]. Direct oral anticoagulants (DOAC) have radically changed anticoagulation management in AF. Indeed, they can be administered in fixed doses without laboratory monitoring and dose adjustment. Even though the introduction of less cumbersome anticoagulant agents has improved, the rates of overall anticoagulant treatment in eligible patients with AF remain to be defined.

Hence, the aim of the study is to evaluate the rates of and determinants for the use of anticoagulation treatment before stroke in patients with known $\mathrm{AF}$ and the adherence to the current treatment guidelines after DOAC availability in clinical practice.

\section{Materials and Methods}

Consecutive patients admitted to the Stroke Unit of the University of Perugia, Italy, from September 2013 through July 2019 for acute ischemic stroke or transient ischemic attack (TIA) with known AF before the event were included in this study. Exclusion criteria were intracranial or subarachnoid hemorrhages and cerebral venous thrombosis.

For all of the included patients the following data were prospectively collected: age, gender, comorbidities (hypertension, diabetes mellitus, hyperlipidemia, and previous ischemic stroke/TIA), NIHSS at admission, current smoker status, obesity and alcoholism. Risk of stroke was defined according to the $\mathrm{CHA}_{2} \mathrm{DS}_{2}$-VASc score [7]. The definition of each piece of collected data has been previously reported [8]. Patients were considered to be adequately treated if they were receiving the following treatment: (1) no treatment in patients with a $\mathrm{CHA}_{2} \mathrm{DS}_{2}$ VASc score of 0 or women with a $\mathrm{CHA}_{2} \mathrm{DS}_{2}$-VASc score of $1,(2)$ oral anticoagulant treatment with VKA with INR between 2 and 3 at time of admission or with DOAC at the labeled dose according to the DOAC summary of product characteristics in patients with a $\mathrm{CHA}_{2} \mathrm{DS}_{2}$-VASc score $>1$ or men with a $\mathrm{CHA}_{2} \mathrm{DS}_{2}$-VASc score of 1 . The outcome of this study was the rate of patients adequately treated according to the current treatment guidelines $[5,6]$.

Categorical data were reported as frequencies and continuous data as means \pm SD. Continuous data were compared using Student's $t$ test whereas categorical data were compared using the $\chi^{2}$ test. The reported $p$ values were based on two-sided tests. Univariate analysis was used to compare clinical data of AF patients treated or not treated with oral anticoagulant therapy before stroke or TIA. Multivariable regression analysis was used to identify independent predictors of anticoagulant treatment before stroke as a dependent variable. The independent variables included in the analysis were: age, sex, history of stroke or TIA, hypertension, diabetes mellitus, hyperlipidemia, and $\mathrm{CHA}_{2} \mathrm{DS}_{2}$-VASc score. Data were analyzed with the SPSS/PC Win package.

\section{Results}

A total of 155 patients with ischemic stroke/TIA and known AF before the event were included in the study. The mean age was $78.8 \pm 8.9$ years and $49 \%$ of the subjects were female. Table 1 shows the main clinical features of the overall patients and patients strat- 

Diseases Extra

Table 1. Characteristics of the study patients

\begin{tabular}{lccclc}
\hline Variable & $\begin{array}{l}\text { Overall } \\
\text { patients } \\
(n=155)\end{array}$ & $\begin{array}{l}\text { No treatment } \\
(n=46)\end{array}$ & $\begin{array}{l}\text { Antiplatelet } \\
\text { therapy } \\
(n=47)\end{array}$ & $\begin{array}{l}\text { Anticoagulant } \\
\text { therapy } \\
(n=62)\end{array}$ & $p$ value $^{\mathrm{a}}$ \\
\hline Age, years & $78.8 \pm 8.9$ & $79.7 \pm 9.0$ & $76.6 \pm 9.2$ & $79.8 \pm 8.4$ & 0.25 \\
Males & $79(51.0)$ & $22(47.8)$ & $24(51.0)$ & $33(53.2)$ & 0.49 \\
NIHSS at admission & $9.1 \pm 6.9$ & $10.7 \pm 6.3$ & $9.0 \pm 7.5$ & $7.9 \pm 6.7$ & 0.20 \\
First-ever stroke & $116(74.8)$ & $39(84.7)$ & $39(82.9)$ & $38(61.2)$ & 0.05 \\
Recurrent stroke & $30(19.4)$ & $6(13.0)$ & $6(12.7)$ & $18(29.0)$ & 0.05 \\
TIA & $9(5.8)$ & $1(2.1)$ & $3(6.3)$ & $5(8.0)$ & 0.70 \\
Hypertension & $122(78.7)$ & $34(73.9)$ & $41(87.2)$ & $47(75.8)$ & 0.59 \\
Diabetes mellitus & $33(21.3)$ & $8(17.3)$ & $14(29.7)$ & $11(17.7)$ & 0.40 \\
Hyperlipidemia & $33(21.3)$ & $7(15.2)$ & $8(17.0)$ & $18(29.0)$ & 0.13 \\
Current smokers & $10(6.5)$ & $1(2.1)$ & $3(6.3)$ & $6(9.6)$ & 0.23 \\
Obesity & $10(6.5)$ & $4(8.6)$ & $4(8.5)$ & $2(3.2)$ & 0.44 \\
Alcoholism & $3(1.9)$ & $3(6.5)$ & 0 & 0 & 0.07 \\
CHA $_{2}$ DS $_{2}$-VASc score $>3$ & $101(65.2)$ & $23(50.0)$ & $32(68.0)$ & $46(74.1)$ & 0.16 \\
\hline
\end{tabular}

Values are presented as means \pm SD or numbers (\%). ${ }^{\text {a }}$ For group comparisons.

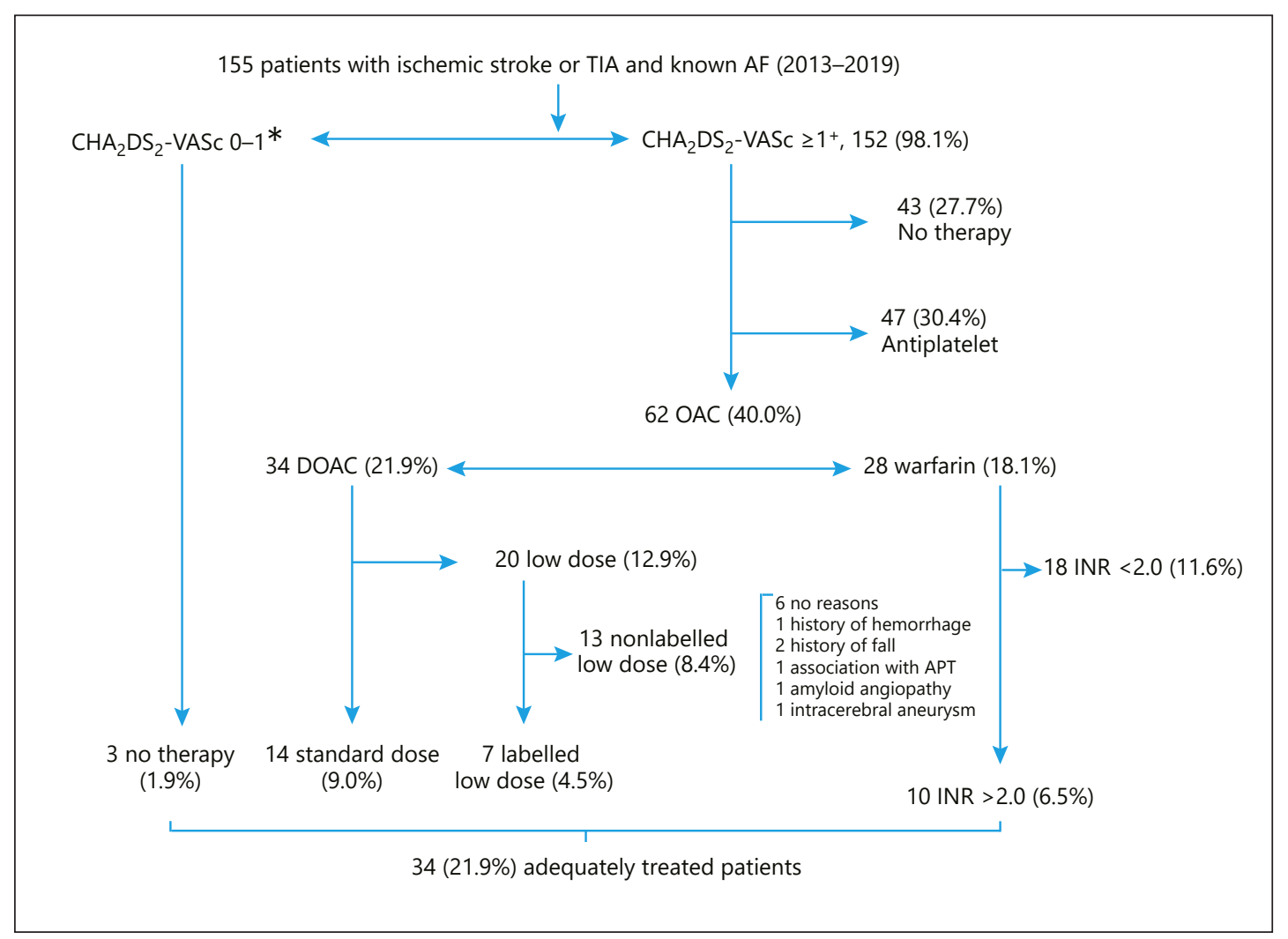

Fig. 1. Flowchart of this study. 


\section{Cerebrovascular Diseases Extra}

\begin{tabular}{l|l}
\hline Cerebrovasc Dis Extra 2020;10:44-49 \\
\hline DOI: 10.1159/000506923 & $\begin{array}{l}\text { @ 2020 The Author(s). Published by S. Karger AG, Basel } \\
\text { www.karger.com/cee }\end{array}$ \\
\hline
\end{tabular}

Giustozzi et al.: Rates and Determinants for the Use of Anticoagulation Treatment before Stroke

Table 2. Characteristics of the patients treated or not with oral anticoagulants and multiple logistic regression with oral anticoagulant therapy as the dependent variable

\begin{tabular}{|c|c|c|c|c|c|c|}
\hline \multirow[t]{2}{*}{ Variable } & \multicolumn{3}{|c|}{ Univariable analysis } & \multicolumn{3}{|c|}{ Multivariable analysis } \\
\hline & $\begin{array}{l}\text { OAC treated } \\
(n=62)\end{array}$ & $\begin{array}{l}\text { not OAC } \\
\text { treated } \\
(n=93)\end{array}$ & $p$ value & OR & $95 \%$ CI & $p$ value \\
\hline Age, years & $79.8 \pm 8.4$ & $78.1 \pm 8.2$ & 0.2 & 1.02 & $0.98-1.06$ & 0.3 \\
\hline Males & $33(53.2)$ & $46(49.5)$ & 0.7 & 1.20 & $0.61-2.39$ & 0.5 \\
\hline NIHSS score at admission & $7.95 \pm 6.79$ & $9.82 \pm 6.98$ & 0.1 & - & - & - \\
\hline History of stroke/TIA & $22(35.3)$ & $14(15.0)$ & 0.03 & 2.93 & $1.29-6.64$ & 0.01 \\
\hline Hypertension & $47(75.8)$ & $75(80.6)$ & 0.5 & 1.01 & $0.43-2.35$ & 0.9 \\
\hline Diabetes mellitus & $11(17.7)$ & $22(23.6)$ & 0.4 & 0.64 & $0.26-1.59$ & 0.3 \\
\hline Hyperlipidemia & $18(29.0)$ & $15(16.1)$ & 0.07 & 2.21 & $0.96-5.08$ & 0.06 \\
\hline Current smokers & $6(9.7)$ & $4(4.3)$ & 0.2 & - & - & - \\
\hline Obesity & $2(3.2)$ & $8(8.6)$ & 0.3 & - & - & - \\
\hline Alcoholism & 0 & $3(3.2)$ & 0.3 & - & - & - \\
\hline $\mathrm{CHA}_{2} \mathrm{DS}_{2}$-VASc $>3$ & $46(74.2)$ & $55(59.1)$ & 0.05 & 1.83 & $0.90-3.78$ & 0.09 \\
\hline Mortality & $2(3.2)$ & $7(7.5)$ & 0.3 & - & - & - \\
\hline
\end{tabular}

Values are presented as means \pm SD or numbers (\%). OAC, oral anticoagulation.

ified by type of treatment received. The majority of them were hospitalized for first-ever ischemic stroke (74.8\%), 30 out of 155 patients were hospitalized for recurrent stroke (19.4\%), and the remaining 5.8\% had a TIA. Hypertension was the most common risk factor for stroke.

Before stroke, only 3 patients had a $\mathrm{CHA}_{2} \mathrm{DS}_{2}$-VASc score of $0-1$ and were not receiving proper treatment (Fig. 1). A CHA $\mathrm{DS}_{2}$-VASc score $>1$ was observed in 152 patients $(98.1 \%)$. Of them, 47 patients were receiving antiplatelet agents and 43 patients were not receiving any treatment. Of 152 patients, $62(40.7 \%)$ were on treatment with oral anticoagulants. Specifically, 34 patients were on DOAC and 28 patients were on VKA. Thirteen patients on DOAC (38.2\%) were receiving a nonlabeled reduced dose and 18 patients on VKA (64.3\%) had an INR value $<2$ at admission. Overall, only 34 out of 155 patients (21.9\%) were adequately treated according to current guidelines before stroke (Fig. 1). The characteristics of the patients treated or not with oral anticoagulants and the factors associated with the use of anticoagulant treatment are listed in Table 2. Previous stroke or TIA was the only independent predictor associated with the use of anticoagulant therapy in patients with AF (OR 2.93; 95\% CI 1.29-6.64). After stroke, no difference in terms of mortality was observed between patients treated or not with oral anticoagulants.

\section{Discussion}

In our study, only $21.9 \%$ of the patients with known AF were adequately treated according to recent guidelines before a stroke or TIA. Previous stroke or TIA was the only independent predictor of the use of anticoagulant therapy in patients with AF.

Anticoagulation use in AF-eligible patients has been reported to have increased since DOAC became available in clinical practice. In a recent study of $655,000 \mathrm{AF}$ patients with a $\mathrm{CHA}_{2} \mathrm{DS}_{2}$-VASc score $>1$, the rate of use of overall anticoagulation increased from 52.4 to $60.7 \%$ following the introduction of DOAC [9]. In our study, we specifically focused on 
patients hospitalized for acute ischemic stroke or TIA with known AF before the event. We observed that only a quarter of AF patients adequately received oral anticoagulation before stroke. We believe that the high proportion of patients not receiving appropriate therapy observed in our study may depend on a bias of patient selection, as having a stroke is often the result of inappropriate therapy. These findings confirm that underuse or premature discontinuation of anticoagulants is a persistent problem. Their predictable effects without monitoring, their ease of use, and their improved safety profile are some of the advantages of DOAC over VKA that can facilitate patient adherence and increase physician confidence in anticoagulant prescription. Indeed, we cannot exclude that, having included in our study patients from 2013 to 2019, the proportion of patients receiving appropriate therapy may have increased over time. Among patients receiving a reduced dose of DOAC, we found that $65 \%$ of the patients were receiving a nonlabeled DOAC reduced dose. There was no indication in 6 out of 13 patients (46\%) had of those patients. Inappropriate DOAC prescription is frequent and it is associated with an increased risk of thromboembolic or bleeding events [10]. Underdosing of DOAC is the most common drug-related problem when using the summaries of product characteristics as a reference. In our view, the reasons for underdosing of DOAC are complex. Overall, physicians tend to underdose anticoagulation due to an excessive fear of bleeding in patients with potential contraindications. In our study the only factor that correlated with the likelihood of being anticoagulated was a previous stroke or TIA. This data reflects the reluctance of physicians to prescribe anticoagulation as primary prevention or in patients without additional risk factors. Even a $\mathrm{CHA}_{2} \mathrm{DS}_{2}$-VASc score $>3$ could be considered a predictor of the use of anticoagulant therapy in patients with $\mathrm{AF}$, despite it being not significant in our study because of the limited simple size. Finally, the limitations of our study were its small sample size and its single-center nature, which may limit the generalizability of our results.

\section{Conclusion}

Only $21.9 \%$ of patients hospitalized for a stroke or TIA with known AF were adequately treated according to recent treatment guidelines. After the introduction of DOAC in clinical practice, the number of AF patients who were adequately treated appears to have increased but it is still unsatisfactory. Therefore, it is important not only to improve medical information about the risk of AF and the efficacy of anticoagulants in stroke prevention but also to support an adequate therapy management. Indeed, the creation of a network between hospitals and general practitioners or the increase in the number of physicians eligible to prescribe anticoagulant therapy may be possible strategies to improve the proper use of anticoagulation in patients with AF.

\section{Statement of Ethics}

This study was approved by the ethical committee and institutional review boards of our institution. Informed consent was provided by the study participants.

\section{Disclosure Statement}

All of the authors have no conflict of interests to declare.

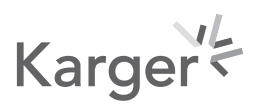




\section{Cerebrovascular \\ Diseases Extra}

\begin{tabular}{l|l}
\hline Cerebrovasc Dis Extra 2020;10:44-49 \\
\hline DOI: 10.1159/000506923 & $\begin{array}{l}\text { ○ } 2020 \text { The Author(s). Published by S. Karger AG, Basel } \\
\text { www.karger.com/cee }\end{array}$ \\
\hline
\end{tabular}

Giustozzi et al.: Rates and Determinants for the Use of Anticoagulation Treatment before Stroke

\section{Funding Sources}

No financial support was received for this study.

\section{Author Contributions}

Michela Giustozzi contributed to the interpretation of data and drafting and critical revision of this paper and is the guarantor of this paper, taking responsibility for the integrity of the work as a whole, from its inception to the published article. Giancarlo Agnelli and Maurizio Paciaroni contributed to the conception and design of this study, to the interpretation of data, and to drafting and critical revision of this paper. Silvia Quattrocchi contributed to the collection of data and to creation of the database of patients included in this study. Monica Acciarresi, Andrea Alberti, Valeria Caso, Maria Cristina Vedovati, and Michele Venti contributed to the drafting and critical revision of this paper.

\section{References}

1 Center for Disease Control and Prevention. https://www.cdc.gov/stroke/. Accessed September 23, 2019.

2 Atrial Fibrillation Investigators. Risk factors for stroke and efficacy of antithrombotic therapy in atrial fibrillation. Analysis of pooled data from five randomized controlled trials. Arch Intern Med. 1994 Jul;154(13): 1449-57.

3 Petersen P, Boysen G, Godtfredsen J, Andersen ED, Andersen B. Placebo-controlled, randomised trial of warfarin and aspirin for prevention of thromboembolic complications in chronic atrial fibrillation. The Copenhagen AFASAK study. Lancet. 1989 Jan;1(8631):175-9.

4 Hart RG, Pearce LA, Aguilar MI. Meta-analysis: antithrombotic therapy to prevent stroke in patients who have nonvalvular atrial fibrillation. Ann Intern Med. 2007 Jun;146(12):857-67.

5 January CT, Wann LS, Calkins H, Chen LY, Cigarroa JE, Cleveland JC Jr, et al. 2019 AHA/ACC/HRS focused update of the 2014 AHA/ACC/HRS guideline for the management of patients with atrial fibrillation: a report of the American College of Cardiology/American Heart Association Task Force on Clinical Practice Guidelines and the Heart Rhythm Society. J Am Coll Cardiol. 2019 Jul;74(1):104-32.

6 Kirchhof P, Benussi S, Kotecha D, Ahlsson A, Atar D, Casadei B, et al.; ESC Scientific Document Group. 2016 ESC Guidelines for the management of atrial fibrillation developed in collaboration with EACTS. Eur Heart J. 2016 Oct;37(38):2893-962.

7 Lip GY, Nieuwlaat R, Pisters R, Lane DA, Crijns HJ. Refining clinical risk stratification for predicting stroke and thromboembolism in atrial fibrillation using a novel risk factor-based approach: the euro heart survey on atrial fibrillation. Chest. $2010 \mathrm{Feb}$;37(2):263-72.

8 Paciaroni M, Agnelli G, Caso V, Venti M, Milia P, Silvestrelli G, et al. Atrial fibrillation in patients with first-ever stroke: frequency, antithrombotic treatment before the event and effect on clinical outcome. J Thromb Haemost. 2005 Jun;3(6):1218-23.

9 Marzec LN, Wang J, Shah ND, Chan PS, Ting HH, Gosch KL, et al. Influence of Direct Oral Anticoagulants on Rates of Oral Anticoagulation for Atrial Fibrillation. J Am Coll Cardiol. 2017 May;69(20):2475-84.

10 Steinberg BA, Shrader P, Thomas L, Ansell J, Fonarow GC, Gersh BJ, et al.; ORBIT-AF Investigators and Patients. Off-Label Dosing of Non-Vitamin K Antagonist Oral Anticoagulants and Adverse Outcomes: the ORBIT-AF II Registry. J Am Coll Cardiol. 2016 Dec;68(24):2597-604. 